CRYSTALLOGRAPHIC COMMUNICATIONS

ISSN 2056-9890

Received 22 March 2019

Accepted 9 April 2019

Edited by A. J. Lough, University of Toronto, Canada

Keywords: crystal structure; 1,3-thiazole; Hirshfeld Surface; hydrogen bonds; $I \ldots I$ and I...S interactions.

CCDC reference: 1908908

Supporting information: this article has supporting information at journals.iucr.org/e

\section{Crystal structure and Hirshfeld surface analysis of $\mathrm{N}$-(5-iodo-4-phenylthiazol-2-yl)acetamide}

\author{
Angel D. Herrera-España, ${ }^{a}$ Jesús Aguilera-González, ${ }^{b}$ Gonzalo J. Mena-Rejón,b \\ Simón Hernández-Ortega ${ }^{c}$ and David Cáceres-Castillo ${ }^{b *}$
}

${ }^{a}$ Centro de Investigaciones Químicas, Instituto de Investigación en Ciencias Básicas y Aplicadas, Universidad Autónoma
del Estado de Morelos, Av. Universidad 1001, Chamilpa, Cuernavaca 62209, Morelos, Mexico, b Facultad de Química,
Universidad Autónoma de Yucatán, Calle 43 No. 613, Col. Inalámbrica, CP 97069, Mérida, Yucatán, Mexico, and
cInstituto de Química, Universidad Nacional Autónoma de México, Circuito Exterior, Ciudad Universitaria, 04510,
Ciudad de México, Mexico. *Correspondence e-mail: david.caceres@uady.mx

Two crystallographically independent molecules $(A$ and $B)$ are present in the asymmetric unit of the title compound, $\mathrm{C}_{11} \mathrm{H}_{9} \mathrm{IN}_{2} \mathrm{OS}$, which differ mainly in the dihedral angle between the phenyl and thiazole rings [38.94 (16) and $32.12(15)^{\circ}$, respectively]. In the crystal, the molecules form $\cdots A \cdots B \cdots A \cdots B \cdots$ chains along the [001] and [010] directions through moderate $\mathrm{N}-\mathrm{H} \cdots \mathrm{O}$ hydrogen bonds and $\mathrm{C}-\mathrm{H} \cdots \pi$ interactions, respectively. The overall three-dimensional network is formed by I $\cdots I$ and I $\cdots S$ interactions. Hirshfeld surface analysis indicates that the most important contributions to the crystal packing are from $\mathrm{H} \cdots \mathrm{C} / \mathrm{C} \cdots \mathrm{H}(26.2 \%), \mathrm{H} \cdots \mathrm{H}(20.9 \%), \mathrm{H} \cdots \mathrm{I} / \mathrm{I} \cdots \mathrm{H}(19.4 \%)$ and $\mathrm{H} \cdots \mathrm{O} / \mathrm{O} \cdots \mathrm{H}$ $(6.8 \%)$ interactions.

\section{Chemical context}

The 1,3-thiazole ring is a structural motif frequently found in the pharmaceutical field in antibacterial (Alam et al., 2014), antifungal (Yu et al., 2007) and antiviral (Liu et al., 2011) agents among others. In the chemotherapy of protozoal diseases, 5-bromo-2-aminothiazole derivatives have been investigated as privileged structures in biological tests against intestinal parasites such as Giardia (Mocelo-Castell et al., 2015). Halo-1,3-thiazole derivatives have proven to be suitable substrates in oxidative addition reactions in the presence of palladium (Wang et al., 2015; Hämmerle et al., 2010). The presence of halogens in the core of thiazole derivatives opens the door to using them as suitable substrates for coupling reactions and to expand the therapeutic potential of a compound by improving the pharmaceutical properties. Transition-metal-catalysed reactions constitute one of the most important and attractive research areas in academia, as well as in the pharmaceutical and fine chemical industries (Zhao et al., 2017; Jana et al., 2011). Cross-coupling reactions usually require, in addition to a transition metal, that the electrophilic coupling partner possesses leaving groups such as $\mathrm{Br}^{-}$or $\mathrm{I}^{-}$among others. The development of suitable halo-1,3thiazole substrates for cross-coupling reactions allows us to report the crystal structure and the Hirshfeld surface analysis of $N$-(5-iodo-4-phenylthiazol-2-yl)acetamide.

\section{Structural commentary}

The title 2-acetoamidothiazole derivative crystallizes in the monoclinic space group $P 2_{1} / c$ with two crystallographically 
independent molecules in the asymmetric unit (Fig. 1). The principal difference between these molecules is the dihedral angle between the phenyl and thiazole rings. In molecule $A$, the thiazole ring (S1/N2/C3-C5) makes a dihedral angle of $38.94(16)^{\circ}$ with the adjacent phenyl ring (C6-C11) while for molecule $B$ the dihedral angle between the S2/N4/C14-C16 and $\mathrm{C} 17-\mathrm{C} 22$ rings is $32.12(15)^{\circ}$. Unlike the related compound 2-acetamido-4-p-tolyl-1,3-thiazole (Lynch et al., 2004) in which the molecule is essentially flat, the presence of the iodine atom at $\mathrm{C} 5$ or $\mathrm{C} 16$ of the title compound induces rotation of the phenyl group attached to the thiazole ring, as also observed in some bromine-substituted phenylthiazole compounds (see the Database survey).

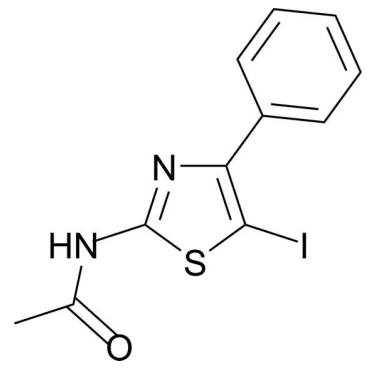

\section{Supramolecular features}

In the crystal, molecules are linked by $\mathrm{N} 1-\mathrm{H} 1 \cdots \mathrm{O} 2$ and $\mathrm{N} 3-$ H3...O1 moderate hydrogen bonds via a C(4) synthon (Table 1, Fig. 2), forming chains along [001] in an $\cdots A \cdots B \cdots A \cdots B \cdots$ fashion. In the same way, the phenyl rings of molecules $A$ and $B$ interact through $\mathrm{C}-\mathrm{H} \cdots \pi$ contacts along [010] and the resulting chains are further connected through $\mathrm{I} 1 \cdots \mathrm{S} 2(1-x,-y, 1-z)$ contacts [3.7758 (9) §] (Fig. 3). Additionally, adjacent $B$ molecules are linked by $\mathrm{I} 2 \cdots \mathrm{I} 2(7-x, 1-y, 1-z)$ contacts of type $\mathrm{I}\left[\theta_{1}=\theta_{2}\right.$ $\left.=146.91(8)^{\circ}\right]$ with a length of 3.8547 (5) $\AA$.

\section{Hirshfeld surface analysis and two-dimensional fingerprints plots}

A Hirshfeld surface analysis was carried out using Crystal Explorer17.5 (Turner et al., 2017) in order to acquire a better

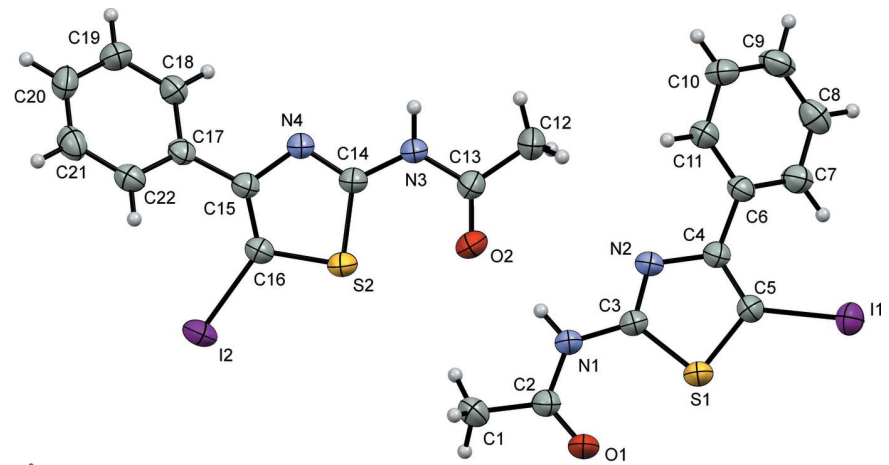

Figure 1

Molecular structure of the two crystallographically independent molecules in the asymmetric unit of the title compound, with the atom labelling. Displacement ellipsoids are drawn at the $50 \%$ probability level and $\mathrm{H}$ atoms are shown as spheres of arbitrary radius.
Table 1

Hydrogen-bond geometry $\left(\AA,^{\circ}\right)$.

$\mathrm{Cg} 2$ and $\mathrm{Cg} 4$ are the centroids of the $\mathrm{C} 6-\mathrm{C} 11$ and $\mathrm{C} 17-\mathrm{C} 22$ rings, respectively.

\begin{tabular}{lllll}
\hline$D-\mathrm{H} \cdots A$ & $D-\mathrm{H}$ & $\mathrm{H} \cdots A$ & $D \cdots A$ & $D-\mathrm{H} \cdots A$ \\
\hline $\mathrm{N} 1-\mathrm{H} 1 \cdots \mathrm{O} 2$ & $0.89(3)$ & $2.03(3)$ & $2.914(3)$ & $175(3)$ \\
$\mathrm{N} 3-\mathrm{H} 3 \cdots \mathrm{O} 1^{\mathrm{i}}$ & $0.89(2)$ & $2.03(2)$ & $2.902(3)$ & $167(2)$ \\
$\mathrm{C} 8-\mathrm{H} 8 \cdots C g 4^{\mathrm{ii}}$ & 0.93 & 2.94 & $3.655(4)$ & 134 \\
$\mathrm{C} 18-\mathrm{H} 18 \cdots C g 2^{\mathrm{ii}}$ & 0.93 & 2.82 & $3.594(4)$ & 141
\end{tabular}

Symmetry codes: (i) $x,-y+\frac{1}{2}, z-\frac{1}{2}$; (ii) $-x+1, y+\frac{1}{2},-z+\frac{1}{2}$.

understanding of the nature of the intermolecular interactions in the title compound. The Hirshfeld surface was generated using a standard (high) surface resolution with the three-

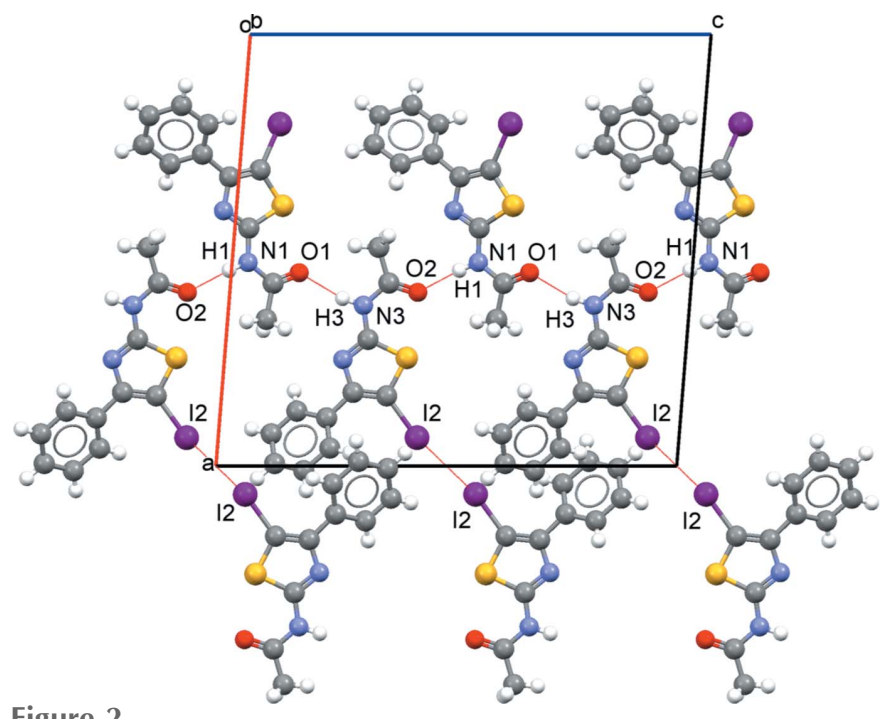

Figure 2

Part of the crystal structure of the title compound, showing the formation of hydrogen bonds and I - I I contacts (red dashed lines) in the $a c$ plane.

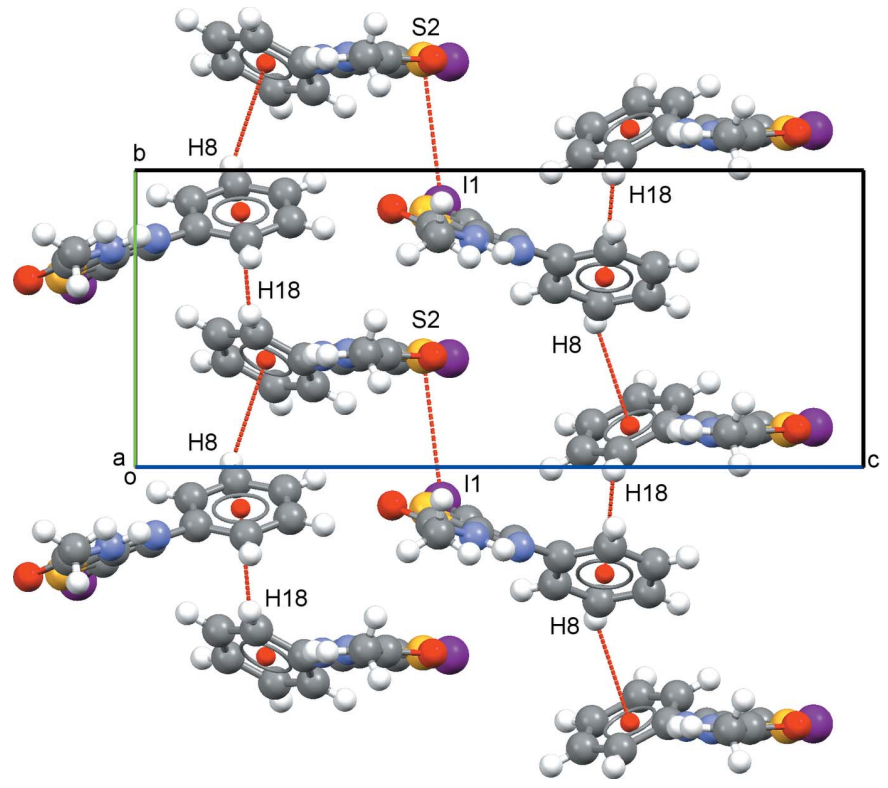

Figure 3

Packing viewed along the $a$-axis direction showing $\mathrm{C}-\mathrm{H} \cdots \pi$ and $\mathrm{I} \cdots \mathrm{S}$ interactions as red dashed lines. 


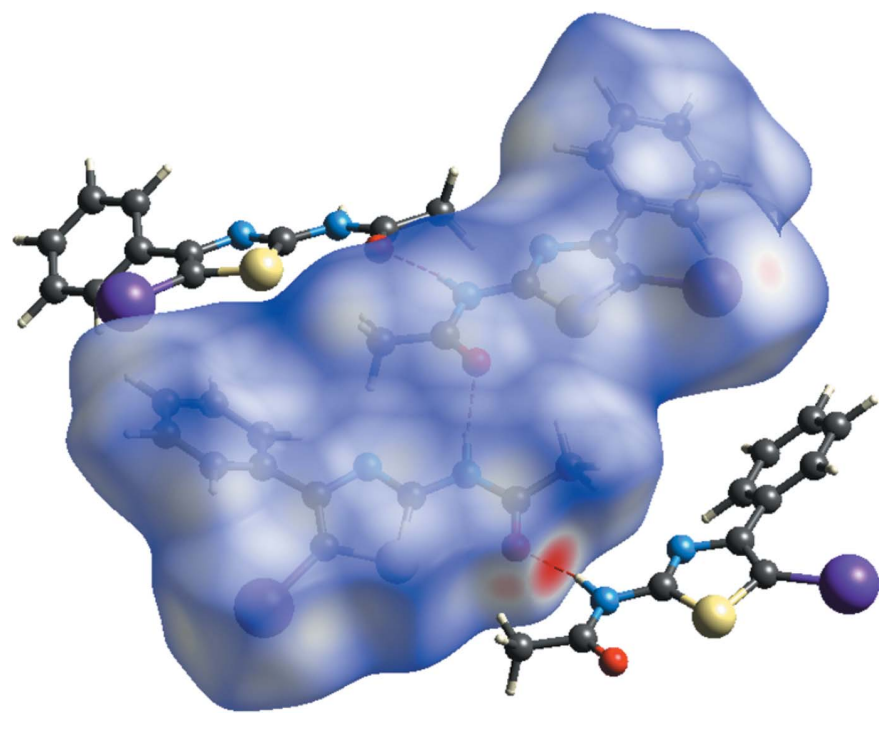

Figure 4

The three-dimensional Hirshfeld surface of the title compound mapped over $d_{\text {norm }}$, showing the $\mathrm{N}-\mathrm{H} \cdots \mathrm{O}$ hydrogen bonds.

dimensional $d_{\text {norm }}$ surface mapped over a fixed color scale of -0.5372 (red) to 1.3937 (blue) a.u. (Fig. 4). The intense red spots on the surface are due to the $\mathrm{N}-\mathrm{H}$. . O hydrogen bonds, resulting from the interaction of the amide group of the 2 -acetoamidothiazole derivative. The overall two-dimensional fingerprint plot for the title compound is shown in Fig. 5a, and those delineated into $\mathrm{H} \cdots \mathrm{C} / \mathrm{C} \cdots \mathrm{H}, \mathrm{H} \cdots \mathrm{H}, \mathrm{H} \cdots \mathrm{I} / \mathrm{I} \cdots \mathrm{H}$, $\mathrm{H} \cdots \mathrm{O} / \mathrm{O} \cdots \mathrm{H}$ and $\mathrm{I} \cdots \mathrm{S} / \mathrm{S} \cdot \mathrm{I}$ contacts are shown in Fig. 5b-f. The major contribution to the crystal packing is from $\mathrm{H} \cdots \mathrm{C} /$ C. . H interactions $(26.2 \%)$. The pair of characteristic wings in this fingerprint plot corresponds to the $\mathrm{C}-\mathrm{H} \cdots \pi$ interactions between the phenyl groups (Fig. $5 b$ ). The $\mathrm{H} \cdots \mathrm{H}$ and $\mathrm{H} \cdots \mathrm{I} /$ I $\cdots H$ contacts (Fig. $5 c$ and $5 d$ ) make similar contributions to

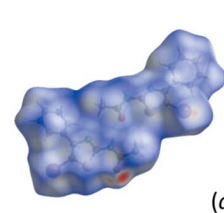

(a)
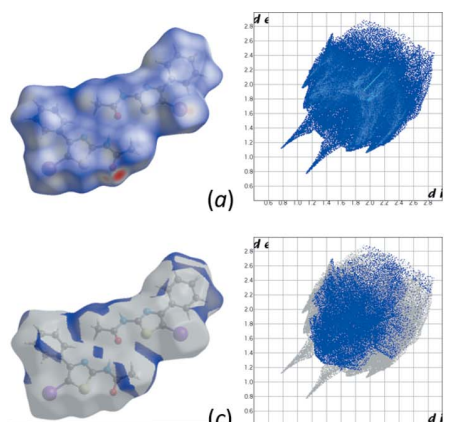

(c)
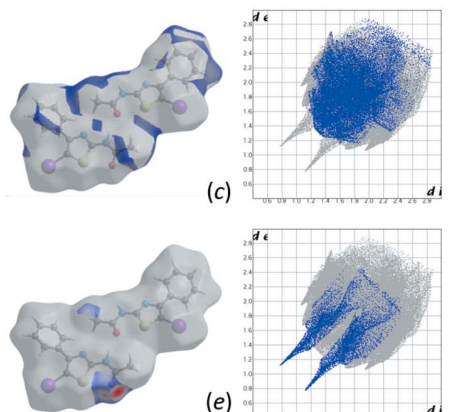

(e)

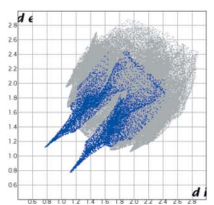

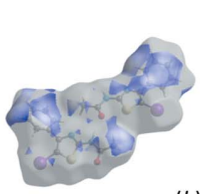

(b)
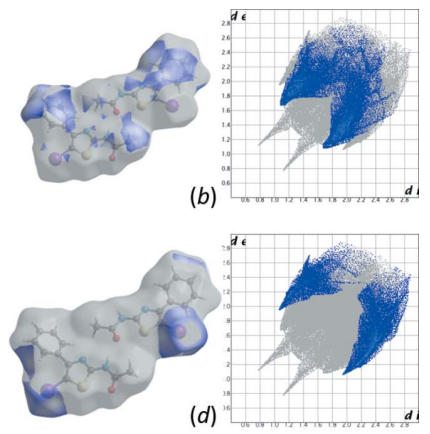

(d)
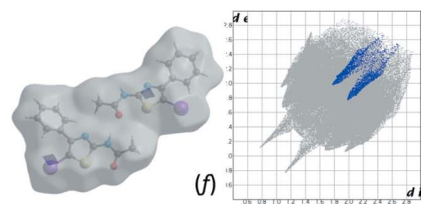

Figure 5

Two-dimensional fingerprint plots for the (a) all, (b) $\mathrm{H} \cdots \mathrm{C} / \mathrm{C} \cdot \mathrm{H}$ $(26.2 \%),(c) \mathrm{H} \cdots \mathrm{H}(20.9 \%),(d) \mathrm{H} \cdots \mathrm{I} / \mathrm{I} \cdots \mathrm{H}(19.4 \%),(e) \mathrm{H} \cdots \mathrm{O} / \mathrm{O} \cdots \mathrm{H}$ $(6.8 \%)$ and $(f) \mathrm{I} \cdots \mathrm{S} / \mathrm{S} \cdots \mathrm{I}(2.2 \%)$ contacts in the title compound. the total Hirshfeld surface of 20.9 and $19.4 \%$, respectively. The reciprocal $\mathrm{H} \cdots \mathrm{O} / \mathrm{O} \cdots \mathrm{H}$ interactions $(6.8 \%)$ are seen as sharp symmetrical spikes with tips at $d_{e}+d_{i} \sim 1.9 \AA$ and arising from the $\mathrm{N}-\mathrm{H} \cdots \mathrm{O}$ hydrogen bond (Fig. $5 e$ ). Intermolecular I $\cdots \mathrm{S} /$ S...I (Fig. 5f) and I...I interactions make smaller contributions to the Hirshfeld surface (2.2 and 1.1\%, respectively).

\section{Database survey}

A search of the Cambridge Structural Database (CSD, Version 5.39, update of February 2018; Groom et al., 2016) for a 1,3 thiazole ring with a benzene ring and a halogen as substituents in positions 4 and 5, respectively, gave four entries for the organobromine compounds $N$-[5-bromo-4-(4-methylphenyl)1,3-thiazol-2-yl]-4-chlorobutanamide (CCDC 1443533; Ghabbour et al., 2016), 5,5'-dibromo-4,4'-bis(pentafluorophenyl)-2,2'-bi-1,3-thiazole (CCDC 889644; Siram et al., 2013), 1-(5-bromo-4-phenyl-1,3-thiazol-2-yl)pyrrolidin-2-one (CCDC 886962; Ghabbour, Kadi, et al., 2012) and 5-bromo-4(3,4-dimethoxyphenyl)-1,3-thiazol-2-amine (CCDC 886876; Ghabbour, Chia, et al., 2012). The dihedral angle between the thiazole and benzene rings in these compounds are in the range $36.69(11)$ to $60.83(3)^{\circ}$, with exception of $N$-(5-bromo4-(4-methylphenyl)-1,3-thiazol-2-yl)-4-chlorobutanamide. In this compound the dihedral angle is smaller $\left[8.8(3)^{\circ}\right]$ as a result of an intramolecular $\mathrm{C}-\mathrm{H} \cdots \mathrm{Br}$ hydrogen bond. In the crystals of these compounds, only 5,5'-dibromo-4,4'-bis(pentafluorophenyl)-2,2'-bi-1,3-thiazole exhibits a type II halogen-halogen interaction with a $\mathrm{Br} \cdots \mathrm{Br}$ distance of 3.6777 (3) $\AA$ and angles of 68.88 (5) and $174.77(5)^{\circ}$.

\section{Synthesis and crystallization}

A mixture of $N$-(4-phenylthiazol-2-yl) acetamide $(0.5 \mathrm{mmol}$, $109 \mathrm{mg}, 1 \mathrm{eq})$ and iodine ( $1 \mathrm{mmol}, 127 \mathrm{mg}, 2 \mathrm{eq})$ was placed in an open vessel containing a Teflon-coated stir bar. The mixture was dissolved in $3 \mathrm{~mL}$ of ethanol and the vessel was placed in the microwave cavity (CEM, Discover) and subjected to MW irradiation $(150 \mathrm{~W})$ for $60 \mathrm{~min}$, at $363 \mathrm{~K}$ and a pressure of 2 psi. The reaction mixture was then cooled at room temperature and $5 \mathrm{~mL}$ of $\mathrm{NH}_{4} \mathrm{OH}$ were added. The obtained mixture was dissolved in ethyl acetate $(50 \mathrm{~mL})$ and washed with brine $(3 \times)$. The organic layer was separated, dehydrated with $\mathrm{Na}_{2} \mathrm{SO}_{4}$, and evaporated in vacuo until dryness. The product was purified by flash column chromatography (silica gel, 2-25 $\mu \mathrm{m}$ ) with a mixture of petrol-dichloromethaneacetone (5:3:2). The title compound was obtained as paleyellow needles in $30 \%$ yield $(52.2 \mathrm{mg}, 0.15 \mathrm{mmol})$. A diluted solution of the compound was prepared in hexane and kept on a dry and dark place at room temperature. Crystals were obtained after one week of slow evaporation. Spectroscopic data: ${ }^{1} \mathrm{H}$ NMR (400 MHz, $\left.\mathrm{CDCl}_{3}\right)$ : $11.37(s, 1 \mathrm{H}), 7.80(m, 2 \mathrm{H})$, $7.43(m, 3 \mathrm{H}), 1.62(s, 3 \mathrm{H}) .{ }^{13} \mathrm{C}$ NMR $\left(100 \mathrm{MHz}, \mathrm{CDCl}_{3}\right): 168.8$ $(s), 163.6(s), 151.4(s), 134.5(s), 129.0(d), 128.9(d), 128.7(d)$, $62.4(s) 21.9(c)$. 


\section{Refinement}

Crystal data, data collection and structure refinement details are summarized in Table 2. Hydrogen atoms bonded to $\mathrm{C}$ atoms were positioned geometrically and refined using a riding model: $\mathrm{C}-\mathrm{H}=0.95-1.00 \AA$ with $U_{\text {iso }}(\mathrm{H})=1.2 U_{\text {eq }}(\mathrm{C})$ or $1.5 U_{\text {eq }}(\mathrm{C}-$ methyl $)$.

\section{Acknowledgements}

JAG thanks the Consejo Nacional de Ciencia y Tecnología (CONACYT) for a BSc scholarship.

\section{Funding information}

Funding for this research was provided by: Consejo Nacional de Ciencia y Tecnología (grant No. 290398).

\section{References}

Alam, M. S., Ahmed, J. U. \& Lee, D. (2014). Chem. Pharm. Bull. 62, 1259-1268.

Bruker (2014). APEX2, SAINT and SADABS. Bruker AXS Inc., Madison, Wisconsin, USA.

Ghabbour, H. A. \& Al-Omar, M. A. (2016). Z. Kristallogr. New Cryst. Struct. 231, 859-860.

Ghabbour, H. A., Chia, T. S. \& Fun, H.-K. (2012). Acta Cryst. E68, o1631-o1632.

Ghabbour, H. A., Kadi, A. A., El-Subbagh, H. I., Chia, T. S. \& Fun, H.-K. (2012). Acta Cryst. E68, o1738-o1739.

Groom, C. R., Bruno, I. J., Lightfoot, M. P. \& Ward, S. C. (2016). Acta Cryst. B72, 171-179.

Hämmerle, J., Schnürch, M., Iqbal, N., Mihovilovic, M. D. \& Stanetty, P. (2010). Tetrahedron, 66, 8051-8059.

Jana, R., Pathak, T. P. \& Sigman, M. S. (2011). Chem. Rev. 111, $1417-$ 1492.

Krause, L., Herbst-Irmer, R., Sheldrick, G. M. \& Stalke, D. (2015). J. Appl. Cryst. 48, 3-10.

Liu, Y., Zhang, L., Gong, J., Fang, H., Liu, A., Du, G. \& Xu, W. (2011). J. Enzyme Inhib. Med. Chem. 26, 506-513.

Lynch, D. E. \& McClenaghan, I. (2004). Acta Cryst. C60, o815-o817.

Macrae, C. F., Edgington, P. R., McCabe, P., Pidcock, E., Shields, G. P., Taylor, R., Towler, M. \& van de Streek, J. (2006). J. Appl. Cryst. 39, 453-457.

Mocelo-Castell, R., Villanueva-Novelo, C., Cáceres-Castillo, D., Carballo, R. M., Quijano-Quiñones, R. F., Quesadas-Rojas, M., Cantillo-Ciau, Z., Cedillo-Rivera, R., Moo-Puc, R. E., Moujir, L. M. \& Mena-Rejón, G. J. (2015). Open Chem. 13, 1127-1136.

Sheldrick, G. M. (2015). Acta Cryst. A71, 3-8.
Table 2

Experimental details.

\begin{tabular}{|c|c|}
\hline \multicolumn{2}{|l|}{ Crystal data } \\
\hline Chemical formula & $\mathrm{C}_{11} \mathrm{H}_{9} \mathrm{IN}_{2} \mathrm{OS}$ \\
\hline$M_{\mathrm{r}}$ & 344.16 \\
\hline Crystal system, space group & Monoclinic, $P 2_{1} / c$ \\
\hline Temperature $(\mathrm{K})$ & 298 \\
\hline$a, b, c(\AA)$ & $17.4130(6), 7.5325(3), 18.5443(6)$ \\
\hline$\beta\left(^{\circ}\right)$ & $94.567(1)$ \\
\hline$V\left(\AA^{3}\right)$ & $2424.61(15)$ \\
\hline$Z$ & 8 \\
\hline Radiation type & Мо $K \alpha$ \\
\hline$\mu\left(\mathrm{mm}^{-1}\right)$ & 2.79 \\
\hline Crystal size $(\mathrm{mm})$ & $0.30 \times 0.27 \times 0.09$ \\
\hline \multicolumn{2}{|l|}{ Data collection } \\
\hline Diffractometer & $\begin{array}{l}\text { Bruker D8 Venture } \kappa \text {-geometry } \\
\text { diffractometer 208039-01 }\end{array}$ \\
\hline Absorption correction & $\begin{array}{l}\text { Multi-scan (SADABS; Krause et } \\
\text { al. 2015) }\end{array}$ \\
\hline$T_{\min }, T_{\max }$ & $0.595,0.745$ \\
\hline $\begin{array}{l}\text { No. of measured, independent and } \\
\text { observed }[I>2 \sigma(I)] \text { reflections }\end{array}$ & $21512,4448,3987$ \\
\hline$R_{\text {int }}$ & 0.020 \\
\hline$(\sin \theta / \lambda)_{\max }\left(\AA^{-1}\right)$ & 0.604 \\
\hline \multicolumn{2}{|l|}{ Refinement } \\
\hline$R\left[F^{2}>2 \sigma\left(F^{2}\right)\right], w R\left(F^{2}\right), S$ & $0.029,0.073,1.12$ \\
\hline No. of reflections & 4448 \\
\hline No. of parameters & 297 \\
\hline No. of restraints & 2 \\
\hline $\mathrm{H}$-atom treatment & $\mathrm{H}$-atom parameters constrained \\
\hline$\Delta \rho_{\max }, \Delta \rho_{\min }\left(\mathrm{e} \AA^{-3}\right)$ & $0.97,-0.49$ \\
\hline
\end{tabular}

Computer programs: APEX3 and SAINT (Bruker, 2014), SHELXS2014 (Bruker, 2014), SHELXL2014 (Sheldrick, 2015), Mercury (Macrae et al., 2006), PLATON (Spek, 2009) and publCIF (Westrip, 2010).

Siram, R. B. K., Karothu, D. P., Guru Row, T. N. \& Patil, S. (2013). Cryst. Growth Des. 13, 1045-1049.

Spek, A. L. (2009). Acta Cryst. D65, 148-155.

Turner, M. J., McKinnon, J. J., Wolff, S. K., Grimwood, D. J., Spackman, P. R., Jayatilaka, D. \& Spackman, M. A. (2017). Crystal. Explorer17. The University of Western Australia.

Wang, F., Yang, Z., Liu, Y., Ma, L., Wu, Y., He, L., Shao, M., Yu, K., Wu, W., Pu, Y., Nie, C. \& Chen, L. (2015). Bioorg. Med. Chem. 23, 3337-3350.

Westrip, S. P. (2010). J. Appl. Cryst. 43, 920-925.

Yu, H., Shao, L. \& Fang, J. (2007). J. Organomet. Chem. 692, 991-996.

Zhao, K., Shen, L., Shen, Z. L. \& Loh, T. P. (2017). Chem. Soc. Rev. 46, 586-602. 


\section{supporting information}

Acta Cryst. (2019). E75, 717-720 [https://doi.org/10.1107/S2056989019004791]

Crystal structure and Hirshfeld surface analysis of N-(5-iodo-4-phenylthiazol-2yl)acetamide

Angel D. Herrera-España, Jesús Aguilera-González, Gonzalo J. Mena-Rejón, Simón HernándezOrtega and David Cáceres-Castillo

Computing details

Data collection: APEX3 (Bruker, 2014); cell refinement: APEX3 (Bruker, 2014); data reduction: SAINT (Bruker, 2014); program(s) used to solve structure: SHELXS2014 (Bruker, 2014); program(s) used to refine structure: SHELXL2014 (Sheldrick, 2015); molecular graphics: Mercury (Macrae et al., 2006); software used to prepare material for publication: PLATON (Spek, 2009) and publCIF (Westrip, 2010).

N-(5-Iodo-4-phenylthiazol-2-yl)acetamide

Crystal data

$\mathrm{C}_{11} \mathrm{H}_{9} \mathrm{IN}_{2} \mathrm{OS}$

$M_{r}=344.16$

Monoclinic, $P 2_{1} / c$

$a=17.4130(6) \AA$

$b=7.5325(3) \AA$

$c=18.5443(6) \AA$

$\beta=94.567(1)^{\circ}$

$V=2424.61(15) \AA^{3}$

$Z=8$

$F(000)=1328$

$D_{\mathrm{x}}=1.886 \mathrm{Mg} \mathrm{m}^{-3}$

Mo $K \alpha$ radiation, $\lambda=0.71073 \AA$

Cell parameters from 9666 reflections

$\theta=2.5-25.4^{\circ}$

$\mu=2.79 \mathrm{~mm}^{-1}$

$T=298 \mathrm{~K}$

Prism, colourless

$0.30 \times 0.27 \times 0.09 \mathrm{~mm}$

\section{Data collection}

Bruker D8 Venture $\kappa$-geometry diffractometer 208039-01

Radiation source: micro-focus X-ray source

Detector resolution: 52.0833 pixels $\mathrm{mm}^{-1}$

$\omega$-scans

Absorption correction: multi-scan

(SADABS; Krause et al., 2015)

$T_{\min }=0.595, T_{\max }=0.745$

21512 measured reflections

4448 independent reflections

3987 reflections with $I>2 \sigma(I)$

$R_{\text {int }}=0.020$

$\theta_{\max }=25.4^{\circ}, \theta_{\min }=2.2^{\circ}$

$h=-15 \rightarrow 21$

$k=-9 \rightarrow 9$

$l=-21 \rightarrow 22$

Refinement

Refinement on $F^{2}$

Least-squares matrix: full

$R\left[F^{2}>2 \sigma\left(F^{2}\right)\right]=0.029$

$w R\left(F^{2}\right)=0.073$

$S=1.12$

4448 reflections

297 parameters

2 restraints

Hydrogen site location: mixed $\mathrm{H}$-atom parameters constrained $w=1 /\left[\sigma^{2}\left(F_{\mathrm{o}}^{2}\right)+(0.0357 P)^{2}+2.3197 P\right]$ where $P=\left(F_{\mathrm{o}}^{2}+2 F_{\mathrm{c}}^{2}\right) / 3$

$(\Delta / \sigma)_{\max }=0.002$

$\Delta \rho_{\max }=0.97 \mathrm{e} \AA^{-3}$

$\Delta \rho_{\min }=-0.49 \mathrm{e} \AA^{-3}$ 


\section{Special details}

Geometry. All esds (except the esd in the dihedral angle between two 1.s. planes) are estimated using the full covariance matrix. The cell esds are taken into account individually in the estimation of esds in distances, angles and torsion angles; correlations between esds in cell parameters are only used when they are defined by crystal symmetry. An approximate (isotropic) treatment of cell esds is used for estimating esds involving l.s. planes.

Fractional atomic coordinates and isotropic or equivalent isotropic displacement parameters $\left(\AA^{2}\right)$

\begin{tabular}{|c|c|c|c|c|}
\hline & $x$ & $y$ & $z$ & $U_{\text {iso }} * / U_{\text {eq }}$ \\
\hline I1 & $0.20804(2)$ & $0.11466(3)$ & $0.57958(2)$ & $0.05010(9)$ \\
\hline $\mathrm{I} 2$ & $0.93426(2)$ & $0.36580(3)$ & $0.43149(2)$ & $0.05145(9)$ \\
\hline S1 & $0.39704(5)$ & $0.14260(11)$ & $0.59979(4)$ & $0.04052(18)$ \\
\hline S2 & $0.74959(5)$ & $0.37404(11)$ & $0.39534(4)$ & $0.03938(18)$ \\
\hline $\mathrm{O} 1$ & $0.55222(13)$ & $0.1295(3)$ & $0.64721(12)$ & $0.0505(6)$ \\
\hline $\mathrm{O} 2$ & $0.59773(14)$ & $0.3722(3)$ & $0.40790(12)$ & $0.0505(6)$ \\
\hline N1 & $0.52822(14)$ & $0.2346(4)$ & $0.53438(13)$ & $0.0413(6)$ \\
\hline H1 & $0.5467(19)$ & $0.278(4)$ & $0.4945(12)$ & $0.050^{*}$ \\
\hline $\mathrm{N} 2$ & 0.40840 (14) & 0.2698 (4) & $0.47229(13)$ & $0.0388(6)$ \\
\hline N3 & $0.62765(15)$ & 0.3834 (4) & $0.29243(13)$ & $0.0408(6)$ \\
\hline H3 & $0.612(2)$ & 0.377 (4) & $0.2456(7)$ & $0.049 *$ \\
\hline N4 & $0.75315(14)$ & $0.3745(3)$ & $0.25708(13)$ & $0.0372(6)$ \\
\hline $\mathrm{C} 1$ & $0.66028(18)$ & $0.2219(5)$ & $0.58392(19)$ & $0.0510(8)$ \\
\hline $\mathrm{H} 1 \mathrm{~A}$ & 0.6829 & 0.2837 & 0.6256 & $0.076^{*}$ \\
\hline H1B & 0.6656 & 0.2923 & 0.5414 & $0.076^{*}$ \\
\hline $\mathrm{H} 1 \mathrm{C}$ & 0.6860 & 0.1103 & 0.5792 & $0.076^{*}$ \\
\hline $\mathrm{C} 2$ & $0.57687(17)$ & 0.1901 (4) & $0.59246(16)$ & $0.0393(7)$ \\
\hline $\mathrm{C} 3$ & 0.44875 (17) & 0.2214 (4) & $0.53026(15)$ & $0.0369(6)$ \\
\hline $\mathrm{C} 4$ & $0.33023(16)$ & 0.2505 (4) & $0.47953(15)$ & $0.0350(6)$ \\
\hline $\mathrm{C} 5$ & $0.31384(16)$ & 0.1834 (4) & $0.54453(16)$ & $0.0371(6)$ \\
\hline C6 & $0.27744(17)$ & $0.3026(4)$ & $0.41671(15)$ & $0.0365(6)$ \\
\hline $\mathrm{C} 7$ & $0.20880(19)$ & 0.3934 (4) & $0.42351(18)$ & $0.0433(7)$ \\
\hline $\mathrm{H} 7$ & 0.1925 & 0.4152 & 0.4692 & $0.052 *$ \\
\hline $\mathrm{C} 8$ & $0.16480(19)$ & $0.4514(5)$ & $0.3630(2)$ & $0.0509(8)$ \\
\hline H8 & 0.1190 & 0.5121 & 0.3681 & $0.061^{*}$ \\
\hline C9 & $0.1884(2)$ & $0.4198(5)$ & $0.29482(19)$ & $0.0522(9)$ \\
\hline H9 & 0.1591 & 0.4607 & 0.2541 & $0.063^{*}$ \\
\hline $\mathrm{C} 10$ & $0.2554(2)$ & $0.3275(5)$ & $0.28749(18)$ & $0.0501(8)$ \\
\hline H10 & 0.2710 & 0.3046 & 0.2416 & $0.060^{*}$ \\
\hline C11 & $0.29986(18)$ & 0.2687 (4) & $0.34786(16)$ & $0.0417(7)$ \\
\hline H11 & 0.3450 & 0.2060 & 0.3423 & $0.050^{*}$ \\
\hline C12 & $0.4936(2)$ & $0.3841(7)$ & $0.3156(2)$ & $0.0688(12)$ \\
\hline $\mathrm{H} 12 \mathrm{~A}$ & 0.4668 & 0.2859 & 0.3350 & $0.103 *$ \\
\hline H12B & 0.4900 & 0.3761 & 0.2638 & $0.103^{*}$ \\
\hline $\mathrm{H} 12 \mathrm{C}$ & 0.4707 & 0.4934 & 0.3298 & $0.103 *$ \\
\hline C13 & $0.57608(18)$ & 0.3794 (4) & $0.34378(17)$ & $0.0413(7)$ \\
\hline $\mathrm{C} 14$ & $0.70669(17)$ & 0.3778 (4) & $0.30805(15)$ & $0.0354(6)$ \\
\hline $\mathrm{C} 15$ & $0.82943(17)$ & 0.3668 (4) & $0.28480(15)$ & $0.0338(6)$ \\
\hline $\mathrm{C} 16$ & $0.83746(17)$ & 0.3663 (4) & $0.35831(16)$ & $0.0363(6)$ \\
\hline
\end{tabular}




$\begin{array}{lllll}\mathrm{C} 17 & 0.88862(17) & 0.3640(4) & 0.23203(16) & 0.0340(6) \\ \mathrm{C} 18 & 0.87296(17) & 0.4513(5) & 0.16611(16) & 0.0418(7) \\ \mathrm{H} 18 & 0.8267 & 0.5121 & 0.1570 & 0.050^{*} \\ \mathrm{C} 19 & 0.92545(19) & 0.4480(5) & 0.11460(18) & 0.0494(8) \\ \mathrm{H} 19 & 0.9145 & 0.5069 & 0.0709 & 0.059^{*} \\ \mathrm{C} 20 & 0.99420(19) & 0.3583(5) & 0.1270(2) & 0.0495(8) \\ \mathrm{H} 20 & 1.0293 & 0.3562 & 0.0918 & 0.059^{*} \\ \mathrm{C} 21 & 1.01068(18) & 0.2721(5) & 0.19149(19) & 0.0477(8) \\ \mathrm{H} 21 & 1.0572 & 0.2119 & 0.2000 & 0.057^{*} \\ \mathrm{C} 22 & 0.95846(18) & 0.2741(4) & 0.24411(17) & 0.0404(7) \\ \mathrm{H} 22 & 0.9701 & 0.2152 & 0.2877 & 0.049^{*}\end{array}$

Atomic displacement parameters $\left(\AA^{2}\right)$

\begin{tabular}{|c|c|c|c|c|c|c|}
\hline & $U^{11}$ & $U^{22}$ & $U^{33}$ & $U^{12}$ & $U^{13}$ & $U^{23}$ \\
\hline I1 & $0.03894(13)$ & $0.06520(16)$ & 0.04648 (14) & $-0.01106(9)$ & $0.00551(10)$ & $0.00738(10)$ \\
\hline I2 & $0.04289(14)$ & $0.07225(18)$ & $0.03680(13)$ & $0.00079(10)$ & $-0.01192(9)$ & $-0.00143(10)$ \\
\hline S1 & $0.0370(4)$ & $0.0575(5)$ & 0.0268 (4) & $-0.0013(3)$ & $0.0002(3)$ & $0.0065(3)$ \\
\hline S2 & $0.0389(4)$ & $0.0537(5)$ & $0.0252(4)$ & -0.0033 & $0.0004(3)$ & $-0.0013(3)$ \\
\hline $\mathrm{O} 1$ & $0.0390(12)$ & $0.0830(18)$ & $0.0287(11)$ & $-0.0006(11)$ & $-0.0023(9)$ & $0.0113(10)$ \\
\hline $\mathrm{O} 2$ & $0.0479(13)$ & $0.0750(17)$ & $0.0293(12)$ & $-0.0054(11)$ & $0.0081(10)$ & $-0.0006(10)$ \\
\hline N1 & $0.0318(13)$ & $0.0654(18)$ & $0.0266(13)$ & $-0.0003(12)$ & $0.0010(10)$ & $0.0076(12)$ \\
\hline $\mathrm{N} 2$ & $0.0324(13)$ & $0.0572(16)$ & $0.0261(12)$ & 0.0005 (11) & $-0.0020(10)$ & $0.0031(11)$ \\
\hline N3 & $0.0316(13)$ & $0.0656(18)$ & $0.0249(12)$ & $-0.0035(11)$ & $0.0015(10)$ & $-0.0014(11)$ \\
\hline N4 & $0.0309(13)$ & $0.0530(16)$ & $0.0275(12)$ & $-0.0010(10)$ & $0.0014(10)$ & $-0.0009(10)$ \\
\hline $\mathrm{C} 1$ & $0.0359(17)$ & $0.071(2)$ & $0.0444(18)$ & 0.0009 (16) & $-0.0046(14)$ & 0.0087 (17) \\
\hline $\mathrm{C} 2$ & $0.0363(16)$ & $0.0507(18)$ & $0.0307(15)$ & 0.0033 (14) & $0.0006(12)$ & $-0.0016(13)$ \\
\hline $\mathrm{C} 3$ & $0.0341(15)$ & $0.0496(18)$ & $0.0269(14)$ & $-0.0006(13)$ & $0.0024(12)$ & 0.0015 (12) \\
\hline $\mathrm{C} 4$ & $0.0315(15)$ & $0.0432(16)$ & $0.0300(15)$ & $0.0004(12)$ & $0.0004(11)$ & $-0.0019(12)$ \\
\hline $\mathrm{C} 5$ & $0.0308(15)$ & $0.0481(17)$ & $0.0323(15)$ & $-0.0034(13)$ & $0.0013(12)$ & $0.0022(13)$ \\
\hline C6 & $0.0325(15)$ & $0.0445(17)$ & $0.0313(15)$ & $-0.0051(13)$ & $-0.0044(12)$ & $0.0016(13)$ \\
\hline $\mathrm{C} 7$ & $0.0426(18)$ & $0.0506(19)$ & $0.0360(16)$ & $0.0042(14)$ & $-0.0015(14)$ & $0.0006(13)$ \\
\hline C8 & $0.0389(18)$ & $0.054(2)$ & $0.058(2)$ & $0.0072(15)$ & $-0.0064(15)$ & $0.0037(17)$ \\
\hline $\mathrm{C} 9$ & $0.050(2)$ & $0.063(2)$ & 0.0411 (19) & $-0.0014(17)$ & $-0.0146(15)$ & $0.0079(16)$ \\
\hline $\mathrm{C} 10$ & $0.052(2)$ & $0.067(2)$ & $0.0300(16)$ & $-0.0039(17)$ & $-0.0016(14)$ & $0.0049(15)$ \\
\hline C11 & $0.0345(16)$ & $0.0541(19)$ & $0.0358(16)$ & $-0.0004(14)$ & $-0.0005(13)$ & $0.0053(14)$ \\
\hline $\mathrm{C} 12$ & $0.0346(18)$ & $0.129(4)$ & $0.044(2)$ & $-0.005(2)$ & $0.0073(16)$ & $-0.002(2)$ \\
\hline $\mathrm{C} 13$ & $0.0355(16)$ & $0.0538(19)$ & $0.0353(17)$ & $-0.0041(13)$ & $0.0059(13)$ & $-0.0032(13)$ \\
\hline $\mathrm{C} 14$ & $0.0340(15)$ & $0.0439(17)$ & $0.0279(14)$ & $-0.0019(12)$ & $0.0003(12)$ & $-0.0013(12)$ \\
\hline $\mathrm{C} 15$ & $0.0326(15)$ & $0.0380(16)$ & $0.0302(15)$ & $-0.0005(12)$ & $-0.0012(12)$ & $-0.0020(11)$ \\
\hline $\mathrm{C} 16$ & $0.0328(15)$ & $0.0438(17)$ & $0.0314(15)$ & $-0.0010(12)$ & $-0.0028(12)$ & 0.0004 (12) \\
\hline $\mathrm{C} 17$ & $0.0306(15)$ & $0.0390(16)$ & $0.0316(15)$ & $-0.0027(12)$ & $-0.0017(12)$ & $-0.0064(12)$ \\
\hline $\mathrm{C} 18$ & $0.0328(15)$ & $0.0544(19)$ & $0.0376(16)$ & $0.0050(14)$ & 0.0001 (13) & 0.0007 (14) \\
\hline C19 & $0.0456(19)$ & $0.069(2)$ & $0.0339(16)$ & $0.0018(17)$ & $0.0043(14)$ & $0.0064(16)$ \\
\hline $\mathrm{C} 20$ & $0.0331(17)$ & $0.068(2)$ & $0.048(2)$ & $-0.0023(15)$ & $0.0115(15)$ & $-0.0061(16)$ \\
\hline $\mathrm{C} 21$ & $0.0327(16)$ & $0.056(2)$ & $0.055(2)$ & 0.0065 (14) & 0.0008 (14) & $-0.0093(16)$ \\
\hline $\mathrm{C} 22$ & $0.0375(16)$ & $0.0443(18)$ & $0.0382(16)$ & $0.0021(13)$ & $-0.0050(13)$ & $0.0007(13)$ \\
\hline
\end{tabular}


Geometric parameters $\left(\AA,{ }^{\circ}\right)$

\begin{tabular}{|c|c|c|c|}
\hline $\mathrm{I} 1-\mathrm{C} 5$ & $2.068(3)$ & $\mathrm{C} 7-\mathrm{C} 8$ & $1.378(5)$ \\
\hline $\mathrm{I} 2-\mathrm{C} 16$ & $2.078(3)$ & $\mathrm{C} 7-\mathrm{H} 7$ & 0.9300 \\
\hline $\mathrm{S} 1-\mathrm{C} 5$ & $1.734(3)$ & $\mathrm{C} 8-\mathrm{C} 9$ & $1.381(5)$ \\
\hline $\mathrm{S} 1-\mathrm{C} 3$ & $1.735(3)$ & $\mathrm{C} 8-\mathrm{H} 8$ & 0.9300 \\
\hline $\mathrm{S} 2-\mathrm{C} 16$ & $1.727(3)$ & $\mathrm{C} 9-\mathrm{C} 10$ & $1.374(5)$ \\
\hline $\mathrm{S} 2-\mathrm{C} 14$ & $1.728(3)$ & $\mathrm{C} 9-\mathrm{H} 9$ & 0.9300 \\
\hline $\mathrm{O} 1-\mathrm{C} 2$ & $1.222(4)$ & $\mathrm{C} 10-\mathrm{C} 11$ & $1.382(4)$ \\
\hline $\mathrm{O} 2-\mathrm{C} 13$ & $1.220(4)$ & $\mathrm{C} 10-\mathrm{H} 10$ & 0.9300 \\
\hline $\mathrm{N} 1-\mathrm{C} 2$ & $1.358(4)$ & $\mathrm{C} 11-\mathrm{H} 11$ & 0.9300 \\
\hline $\mathrm{N} 1-\mathrm{C} 3$ & $1.383(4)$ & $\mathrm{C} 12-\mathrm{C} 13$ & $1.490(5)$ \\
\hline $\mathrm{N} 1-\mathrm{H} 1$ & $0.890(10)$ & $\mathrm{C} 12-\mathrm{H} 12 \mathrm{~A}$ & 0.9600 \\
\hline $\mathrm{N} 2-\mathrm{C} 3$ & $1.289(4)$ & $\mathrm{C} 12-\mathrm{H} 12 \mathrm{~B}$ & 0.9600 \\
\hline $\mathrm{N} 2-\mathrm{C} 4$ & $1.386(4)$ & $\mathrm{C} 12-\mathrm{H} 12 \mathrm{C}$ & 0.9600 \\
\hline $\mathrm{N} 3-\mathrm{C} 13$ & $1.360(4)$ & $\mathrm{C} 15-\mathrm{C} 16$ & $1.359(4)$ \\
\hline $\mathrm{N} 3-\mathrm{C} 14$ & $1.384(4)$ & $\mathrm{C} 15-\mathrm{C} 17$ & $1.477(4)$ \\
\hline $\mathrm{N} 3-\mathrm{H} 3$ & $0.891(10)$ & $\mathrm{C} 17-\mathrm{C} 22$ & $1.394(4)$ \\
\hline $\mathrm{N} 4-\mathrm{C} 14$ & $1.292(4)$ & $\mathrm{C} 17-\mathrm{C} 18$ & $1.396(4)$ \\
\hline $\mathrm{N} 4-\mathrm{C} 15$ & $1.387(4)$ & $\mathrm{C} 18-\mathrm{C} 19$ & $1.374(4)$ \\
\hline $\mathrm{C} 1-\mathrm{C} 2$ & $1.493(4)$ & $\mathrm{C} 18-\mathrm{H} 18$ & 0.9300 \\
\hline $\mathrm{C} 1-\mathrm{H} 1 \mathrm{~A}$ & 0.9600 & $\mathrm{C} 19-\mathrm{C} 20$ & $1.378(5)$ \\
\hline $\mathrm{C} 1-\mathrm{H} 1 \mathrm{~B}$ & 0.9600 & $\mathrm{C} 19-\mathrm{H} 19$ & 0.9300 \\
\hline $\mathrm{C} 1-\mathrm{H} 1 \mathrm{C}$ & 0.9600 & $\mathrm{C} 20-\mathrm{C} 21$ & $1.371(5)$ \\
\hline $\mathrm{C} 4-\mathrm{C} 5$ & $1.358(4)$ & $\mathrm{C} 20-\mathrm{H} 20$ & 0.9300 \\
\hline $\mathrm{C} 4-\mathrm{C} 6$ & $1.478(4)$ & $\mathrm{C} 21-\mathrm{C} 22$ & $1.386(4)$ \\
\hline $\mathrm{C} 6-\mathrm{C} 11$ & $1.388(4)$ & $\mathrm{C} 21-\mathrm{H} 21$ & 0.9300 \\
\hline $\mathrm{C} 6-\mathrm{C} 7$ & $1.392(4)$ & $\mathrm{C} 22-\mathrm{H} 22$ & 0.9300 \\
\hline $\mathrm{C} 5-\mathrm{S} 1-\mathrm{C} 3$ & $87.64(14)$ & $\mathrm{C} 9-\mathrm{C} 10-\mathrm{H} 10$ & 119.8 \\
\hline $\mathrm{C} 16-\mathrm{S} 2-\mathrm{C} 14$ & $87.64(14)$ & $\mathrm{C} 11-\mathrm{C} 10-\mathrm{H} 10$ & 119.8 \\
\hline $\mathrm{C} 2-\mathrm{N} 1-\mathrm{C} 3$ & $125.7(3)$ & $\mathrm{C} 10-\mathrm{C} 11-\mathrm{C} 6$ & $120.4(3)$ \\
\hline $\mathrm{C} 2-\mathrm{N} 1-\mathrm{H} 1$ & $120(2)$ & $\mathrm{C} 10-\mathrm{C} 11-\mathrm{H} 11$ & 119.8 \\
\hline $\mathrm{C} 3-\mathrm{N} 1-\mathrm{H} 1$ & $114(2)$ & $\mathrm{C} 6-\mathrm{C} 11-\mathrm{H} 11$ & 119.8 \\
\hline $\mathrm{C} 3-\mathrm{N} 2-\mathrm{C} 4$ & $111.3(2)$ & $\mathrm{C} 13-\mathrm{C} 12-\mathrm{H} 12 \mathrm{~A}$ & 109.5 \\
\hline $\mathrm{C} 13-\mathrm{N} 3-\mathrm{C} 14$ & $123.6(3)$ & $\mathrm{C} 13-\mathrm{C} 12-\mathrm{H} 12 \mathrm{~B}$ & 109.5 \\
\hline $\mathrm{C} 13-\mathrm{N} 3-\mathrm{H} 3$ & $121(2)$ & $\mathrm{H} 12 \mathrm{~A}-\mathrm{C} 12-\mathrm{H} 12 \mathrm{~B}$ & 109.5 \\
\hline $\mathrm{C} 14-\mathrm{N} 3-\mathrm{H} 3$ & $115(2)$ & $\mathrm{C} 13-\mathrm{C} 12-\mathrm{H} 12 \mathrm{C}$ & 109.5 \\
\hline $\mathrm{C} 14-\mathrm{N} 4-\mathrm{C} 15$ & $111.5(2)$ & $\mathrm{H} 12 \mathrm{~A}-\mathrm{C} 12-\mathrm{H} 12 \mathrm{C}$ & 109.5 \\
\hline $\mathrm{C} 2-\mathrm{C} 1-\mathrm{H} 1 \mathrm{~A}$ & 109.5 & $\mathrm{H} 12 \mathrm{~B}-\mathrm{C} 12-\mathrm{H} 12 \mathrm{C}$ & 109.5 \\
\hline $\mathrm{C} 2-\mathrm{C} 1-\mathrm{H} 1 \mathrm{~B}$ & 109.5 & $\mathrm{O} 2-\mathrm{C} 13-\mathrm{N} 3$ & $120.9(3)$ \\
\hline $\mathrm{H} 1 \mathrm{~A}-\mathrm{C} 1-\mathrm{H} 1 \mathrm{~B}$ & 109.5 & $\mathrm{O} 2-\mathrm{C} 13-\mathrm{C} 12$ & $123.9(3)$ \\
\hline $\mathrm{C} 2-\mathrm{C} 1-\mathrm{H} 1 \mathrm{C}$ & 109.5 & $\mathrm{~N} 3-\mathrm{C} 13-\mathrm{C} 12$ & $115.2(3)$ \\
\hline $\mathrm{H} 1 \mathrm{~A}-\mathrm{C} 1-\mathrm{H} 1 \mathrm{C}$ & 109.5 & $\mathrm{~N} 4-\mathrm{C} 14-\mathrm{N} 3$ & $121.2(3)$ \\
\hline $\mathrm{H} 1 \mathrm{~B}-\mathrm{C} 1-\mathrm{H} 1 \mathrm{C}$ & 109.5 & $\mathrm{~N} 4-\mathrm{C} 14-\mathrm{S} 2$ & $115.8(2)$ \\
\hline $\mathrm{O} 1-\mathrm{C} 2-\mathrm{N} 1$ & $120.9(3)$ & $\mathrm{N} 3-\mathrm{C} 14-\mathrm{S} 2$ & $123.0(2)$ \\
\hline $\mathrm{O} 1-\mathrm{C} 2-\mathrm{C} 1$ & $123.9(3)$ & $\mathrm{C} 16-\mathrm{C} 15-\mathrm{N} 4$ & $113.0(3)$ \\
\hline $\mathrm{N} 1-\mathrm{C} 2-\mathrm{C} 1$ & $115.3(3)$ & $\mathrm{C} 16-\mathrm{C} 15-\mathrm{C} 17$ & $130.0(3)$ \\
\hline
\end{tabular}




\begin{tabular}{|c|c|c|c|}
\hline $\mathrm{N} 2-\mathrm{C} 3-\mathrm{N} 1$ & $120.1(3)$ & $\mathrm{N} 4-\mathrm{C} 15-\mathrm{C} 17$ & $117.0(2)$ \\
\hline $\mathrm{N} 2-\mathrm{C} 3-\mathrm{S} 1$ & $115.8(2)$ & $\mathrm{C} 15-\mathrm{C} 16-\mathrm{S} 2$ & $112.0(2)$ \\
\hline $\mathrm{N} 1-\mathrm{C} 3-\mathrm{S} 1$ & $124.0(2)$ & $\mathrm{C} 15-\mathrm{C} 16-\mathrm{I} 2$ & $131.9(2)$ \\
\hline $\mathrm{C} 5-\mathrm{C} 4-\mathrm{N} 2$ & $113.7(3)$ & $\mathrm{S} 2-\mathrm{C} 16-\mathrm{I} 2$ & $116.02(16)$ \\
\hline $\mathrm{C} 5-\mathrm{C} 4-\mathrm{C} 6$ & $129.6(3)$ & $\mathrm{C} 22-\mathrm{C} 17-\mathrm{C} 18$ & $118.5(3)$ \\
\hline $\mathrm{N} 2-\mathrm{C} 4-\mathrm{C} 6$ & $116.7(2)$ & $\mathrm{C} 22-\mathrm{C} 17-\mathrm{C} 15$ & $123.1(3)$ \\
\hline $\mathrm{C} 4-\mathrm{C} 5-\mathrm{S} 1$ & $111.5(2)$ & $\mathrm{C} 18-\mathrm{C} 17-\mathrm{C} 15$ & $118.4(3)$ \\
\hline $\mathrm{C} 4-\mathrm{C} 5-\mathrm{I} 1$ & $128.9(2)$ & $\mathrm{C} 19-\mathrm{C} 18-\mathrm{C} 17$ & $120.4(3)$ \\
\hline $\mathrm{S} 1-\mathrm{C} 5-\mathrm{I} 1$ & $119.55(15)$ & $\mathrm{C} 19-\mathrm{C} 18-\mathrm{H} 18$ & 119.8 \\
\hline $\mathrm{C} 11-\mathrm{C} 6-\mathrm{C} 7$ & $118.7(3)$ & $\mathrm{C} 17-\mathrm{C} 18-\mathrm{H} 18$ & 119.8 \\
\hline $\mathrm{C} 11-\mathrm{C} 6-\mathrm{C} 4$ & $118.2(3)$ & $\mathrm{C} 18-\mathrm{C} 19-\mathrm{C} 20$ & $120.7(3)$ \\
\hline $\mathrm{C} 7-\mathrm{C} 6-\mathrm{C} 4$ & $122.9(3)$ & $\mathrm{C} 18-\mathrm{C} 19-\mathrm{H} 19$ & 119.7 \\
\hline $\mathrm{C} 8-\mathrm{C} 7-\mathrm{C} 6$ & $120.5(3)$ & $\mathrm{C} 20-\mathrm{C} 19-\mathrm{H} 19$ & 119.7 \\
\hline $\mathrm{C} 8-\mathrm{C} 7-\mathrm{H} 7$ & 119.7 & $\mathrm{C} 21-\mathrm{C} 20-\mathrm{C} 19$ & $119.8(3)$ \\
\hline $\mathrm{C} 6-\mathrm{C} 7-\mathrm{H} 7$ & 119.7 & $\mathrm{C} 21-\mathrm{C} 20-\mathrm{H} 20$ & 120.1 \\
\hline $\mathrm{C} 7-\mathrm{C} 8-\mathrm{C} 9$ & $120.2(3)$ & $\mathrm{C} 19-\mathrm{C} 20-\mathrm{H} 20$ & 120.1 \\
\hline $\mathrm{C} 7-\mathrm{C} 8-\mathrm{H} 8$ & 119.9 & $\mathrm{C} 20-\mathrm{C} 21-\mathrm{C} 22$ & $120.4(3)$ \\
\hline $\mathrm{C} 9-\mathrm{C} 8-\mathrm{H} 8$ & 119.9 & $\mathrm{C} 20-\mathrm{C} 21-\mathrm{H} 21$ & 119.8 \\
\hline $\mathrm{C} 10-\mathrm{C} 9-\mathrm{C} 8$ & $119.7(3)$ & $\mathrm{C} 22-\mathrm{C} 21-\mathrm{H} 21$ & 119.8 \\
\hline $\mathrm{C} 10-\mathrm{C} 9-\mathrm{H} 9$ & 120.2 & $\mathrm{C} 21-\mathrm{C} 22-\mathrm{C} 17$ & $120.3(3)$ \\
\hline $\mathrm{C} 8-\mathrm{C} 9-\mathrm{H} 9$ & 120.2 & $\mathrm{C} 21-\mathrm{C} 22-\mathrm{H} 22$ & 119.8 \\
\hline $\mathrm{C} 9-\mathrm{C} 10-\mathrm{C} 11$ & $120.5(3)$ & $\mathrm{C} 17-\mathrm{C} 22-\mathrm{H} 22$ & 119.8 \\
\hline $\mathrm{C} 3-\mathrm{N} 1-\mathrm{C} 2-\mathrm{O} 1$ & $1.3(5)$ & $\mathrm{C} 14-\mathrm{N} 3-\mathrm{C} 13-\mathrm{O} 2$ & $0.7(5)$ \\
\hline $\mathrm{C} 3-\mathrm{N} 1-\mathrm{C} 2-\mathrm{C} 1$ & $-178.3(3)$ & $\mathrm{C} 14-\mathrm{N} 3-\mathrm{C} 13-\mathrm{C} 12$ & $-179.2(3)$ \\
\hline $\mathrm{C} 4-\mathrm{N} 2-\mathrm{C} 3-\mathrm{N} 1$ & $-178.5(3)$ & $\mathrm{C} 15-\mathrm{N} 4-\mathrm{C} 14-\mathrm{N} 3$ & $-179.3(3)$ \\
\hline $\mathrm{C} 4-\mathrm{N} 2-\mathrm{C} 3-\mathrm{S} 1$ & $1.2(4)$ & $\mathrm{C} 15-\mathrm{N} 4-\mathrm{C} 14-\mathrm{S} 2$ & $0.6(3)$ \\
\hline $\mathrm{C} 2-\mathrm{N} 1-\mathrm{C} 3-\mathrm{N} 2$ & $179.2(3)$ & $\mathrm{C} 13-\mathrm{N} 3-\mathrm{C} 14-\mathrm{N} 4$ & $177.1(3)$ \\
\hline $\mathrm{C} 2-\mathrm{N} 1-\mathrm{C} 3-\mathrm{S} 1$ & $-0.5(5)$ & $\mathrm{C} 13-\mathrm{N} 3-\mathrm{C} 14-\mathrm{S} 2$ & $-2.7(4)$ \\
\hline $\mathrm{C} 5-\mathrm{S} 1-\mathrm{C} 3-\mathrm{N} 2$ & $-0.9(3)$ & $\mathrm{C} 16-\mathrm{S} 2-\mathrm{C} 14-\mathrm{N} 4$ & $-0.3(2)$ \\
\hline $\mathrm{C} 5-\mathrm{S} 1-\mathrm{C} 3-\mathrm{N} 1$ & $178.8(3)$ & $\mathrm{C} 16-\mathrm{S} 2-\mathrm{C} 14-\mathrm{N} 3$ & $179.5(3)$ \\
\hline $\mathrm{C} 3-\mathrm{N} 2-\mathrm{C} 4-\mathrm{C} 5$ & $-0.9(4)$ & $\mathrm{C} 14-\mathrm{N} 4-\mathrm{C} 15-\mathrm{C} 16$ & $-0.6(4)$ \\
\hline $\mathrm{C} 3-\mathrm{N} 2-\mathrm{C} 4-\mathrm{C} 6$ & $179.6(3)$ & $\mathrm{C} 14-\mathrm{N} 4-\mathrm{C} 15-\mathrm{C} 17$ & $-179.5(3)$ \\
\hline $\mathrm{N} 2-\mathrm{C} 4-\mathrm{C} 5-\mathrm{S} 1$ & $0.3(4)$ & $\mathrm{N} 4-\mathrm{C} 15-\mathrm{C} 16-\mathrm{S} 2$ & $0.4(3)$ \\
\hline $\mathrm{C} 6-\mathrm{C} 4-\mathrm{C} 5-\mathrm{S} 1$ & $179.6(3)$ & $\mathrm{C} 17-\mathrm{C} 15-\mathrm{C} 16-\mathrm{S} 2$ & $179.1(2)$ \\
\hline $\mathrm{N} 2-\mathrm{C} 4-\mathrm{C} 5-\mathrm{I} 1$ & $-175.6(2)$ & $\mathrm{N} 4-\mathrm{C} 15-\mathrm{C} 16-\mathrm{I} 2$ & $-177.5(2)$ \\
\hline $\mathrm{C} 6-\mathrm{C} 4-\mathrm{C} 5-\mathrm{I} 1$ & $3.7(5)$ & $\mathrm{C} 17-\mathrm{C} 15-\mathrm{C} 16-\mathrm{I} 2$ & $1.3(5)$ \\
\hline $\mathrm{C} 3-\mathrm{S} 1-\mathrm{C} 5-\mathrm{C} 4$ & $0.3(3)$ & $\mathrm{C} 14-\mathrm{S} 2-\mathrm{C} 16-\mathrm{C} 15$ & $0.0(2)$ \\
\hline $\mathrm{C} 3-\mathrm{S} 1-\mathrm{C} 5-\mathrm{I} 1$ & $176.6(2)$ & $\mathrm{C} 14-\mathrm{S} 2-\mathrm{C} 16-\mathrm{I} 2$ & $178.15(17)$ \\
\hline $\mathrm{C} 5-\mathrm{C} 4-\mathrm{C} 6-\mathrm{C} 11$ & $-142.8(3)$ & $\mathrm{C} 16-\mathrm{C} 15-\mathrm{C} 17-\mathrm{C} 22$ & $33.8(5)$ \\
\hline $\mathrm{N} 2-\mathrm{C} 4-\mathrm{C} 6-\mathrm{C} 11$ & $36.5(4)$ & $\mathrm{N} 4-\mathrm{C} 15-\mathrm{C} 17-\mathrm{C} 22$ & $-147.5(3)$ \\
\hline $\mathrm{C} 5-\mathrm{C} 4-\mathrm{C} 6-\mathrm{C} 7$ & $41.2(5)$ & $\mathrm{C} 16-\mathrm{C} 15-\mathrm{C} 17-\mathrm{C} 18$ & $-148.0(3)$ \\
\hline $\mathrm{N} 2-\mathrm{C} 4-\mathrm{C} 6-\mathrm{C} 7$ & $-139.5(3)$ & $\mathrm{N} 4-\mathrm{C} 15-\mathrm{C} 17-\mathrm{C} 18$ & $30.7(4)$ \\
\hline $\mathrm{C} 11-\mathrm{C} 6-\mathrm{C} 7-\mathrm{C} 8$ & $-1.3(5)$ & $\mathrm{C} 22-\mathrm{C} 17-\mathrm{C} 18-\mathrm{C} 19$ & $0.0(5)$ \\
\hline $\mathrm{C} 4-\mathrm{C} 6-\mathrm{C} 7-\mathrm{C} 8$ & $174.7(3)$ & $\mathrm{C} 15-\mathrm{C} 17-\mathrm{C} 18-\mathrm{C} 19$ & $-178.2(3)$ \\
\hline $\mathrm{C} 6-\mathrm{C} 7-\mathrm{C} 8-\mathrm{C} 9$ & $0.1(5)$ & $\mathrm{C} 17-\mathrm{C} 18-\mathrm{C} 19-\mathrm{C} 20$ & $0.2(5)$ \\
\hline $\mathrm{C} 7-\mathrm{C} 8-\mathrm{C} 9-\mathrm{C} 10$ & $1.0(6)$ & $\mathrm{C} 18-\mathrm{C} 19-\mathrm{C} 20-\mathrm{C} 21$ & $-0.3(6)$ \\
\hline $\mathrm{C} 8-\mathrm{C} 9-\mathrm{C} 10-\mathrm{C} 11$ & $-1.0(6)$ & $\mathrm{C} 19-\mathrm{C} 20-\mathrm{C} 21-\mathrm{C} 22$ & $0.3(5)$ \\
\hline
\end{tabular}




$\begin{array}{llll}\mathrm{C} 9-\mathrm{C} 10-\mathrm{C} 11-\mathrm{C} 6 & -0.2(5) & \mathrm{C} 20-\mathrm{C} 21-\mathrm{C} 22-\mathrm{C} 17 & 0.0(5) \\ \mathrm{C} 7-\mathrm{C} 6-\mathrm{C} 11-\mathrm{C} 10 & 1.3(5) & \mathrm{C} 18-\mathrm{C} 17-\mathrm{C} 22-\mathrm{C} 21 & -0.1(4) \\ \mathrm{C} 4-\mathrm{C} 6-\mathrm{C} 11-\mathrm{C} 10 & -174.8(3) & \mathrm{C} 15-\mathrm{C} 17-\mathrm{C} 22-\mathrm{C} 21 & 178.1(3)\end{array}$

Hydrogen-bond geometry $\left(A,{ }^{\circ}\right)$

$C g 2$ and $C g 4$ are the centroids of the $\mathrm{C} 6-\mathrm{C} 11$ and $\mathrm{C} 17-\mathrm{C} 22$ rings, respectively.

\begin{tabular}{lllll}
\hline$D-\mathrm{H} \cdots A$ & $D-\mathrm{H}$ & $\mathrm{H} \cdots A$ & $D \cdots A$ & $D-\mathrm{H} \cdots A$ \\
\hline $\mathrm{N} 1-\mathrm{H} 1 \cdots \mathrm{O} 2$ & $0.89(3)$ & $2.03(3)$ & $2.914(3)$ & $175(3)$ \\
$\mathrm{N} 3-\mathrm{H} 3 \cdots \mathrm{O} 1^{\mathrm{i}}$ & $0.89(2)$ & $2.03(2)$ & $2.902(3)$ & $167(2)$ \\
$\mathrm{C} 8-\mathrm{H} 8 \cdots C g 4^{\mathrm{ii}}$ & 0.93 & 2.94 & $3.655(4)$ & 134 \\
$\mathrm{C} 18-\mathrm{H} 18 \cdots C g 2^{\mathrm{ii}}$ & 0.93 & 2.82 & $3.594(4)$ & 141
\end{tabular}

Symmetry codes: (i) $x,-y+1 / 2, z-1 / 2$; (ii) $-x+1, y+1 / 2,-z+1 / 2$. 